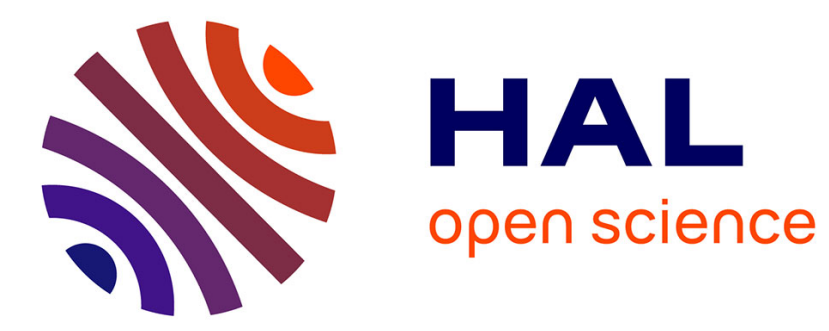

\title{
Utilisation d'un ordinateur associé à des chambres à étincelles
}

\author{
A. Katz, R. Segalas
}

\section{To cite this version:}

A. Katz, R. Segalas. Utilisation d'un ordinateur associé à des chambres à étincelles. Revue de Physique Appliquée, 1969, 4 (2), pp.113-114. 10.1051/rphysap:0196900402011300 . jpa-00243169

\section{HAL Id: jpa-00243169 https://hal.science/jpa-00243169}

Submitted on 1 Jan 1969

HAL is a multi-disciplinary open access archive for the deposit and dissemination of scientific research documents, whether they are published or not. The documents may come from teaching and research institutions in France or abroad, or from public or private research centers.
L'archive ouverte pluridisciplinaire HAL, est destinée au dépôt et à la diffusion de documents scientifiques de niveau recherche, publiés ou non, émanant des établissements d'enseignement et de recherche français ou étrangers, des laboratoires publics ou privés. 


\title{
UTILISATION D’UN ORDINATEUR ASSOGIÉ A DES GHAMBRES A ÉTINGELLES
}

\author{
A. KATZ et R. SEGALAS, \\ Centre d'Études Nucléaires de Saclay.
}

\begin{abstract}
Résumé. - On décrit d'abord très sommairement l'expérience de physique et son équipement, ainsi que l'ordinateur qui constitue le cœur du système mis " en ligne ". Une seconde partie décrit rapidement les moyens originaux mis en œuvre, tant sur le plan de l'électronique d'interface que sur celui de la programmation.
\end{abstract}

Abstract. - First, we describe briefly the physics experiment with its equipments and the computer which is the heart of the on-line system. The second part describes the methods used for the electronics as well as for programming the experiment.

序

L'expérience de physique corpusculaire à haute énergie, qui se déroule actuellement à Saturne, vise à analyser l'interaction $\pi$-nucléon en étudiant la diffusion élastique vers l'arrière de mésons $\pi$ sur des protons $\left({ }^{1}\right)$. Elle associe, à un ensemble expérimental composé de huit chambres à étincelles du type à fils et de compteurs divers, un ordinateur CII 90-40 à qui sont confiés l'acquisition et l'enregistrement des différentes informations de physique, la surveillance et la gestion de l'expérience, et le dialogue avec l'expérimentateur. Parmi ces différentes tâches, ce sont celles d'acquisition des informations et celles de gestion et de dialogue qui posent des problèmes particulièrement intéressants. Les solutions retenues, tant sur le plan de l'électronique d'interface que sur celui de la programmation en temps réel, sont rapidement exposées ci-dessous après une brève description du système installé à Saturne.

I. Le système. - Il est construit autour de l'ordinateur CII 90-40 constitué par une mémoire de 16 kmots de 24 digits (en deux blocs), d'une unité centrale au cycle de base de $1,75 \mu \mathrm{s}$, de deux machines à écrire - dont une installée dans l'aire expérimentale d'une imprimante rapide, d'un lecteur perforateur de ruban, d'un lecteur de cartes et de trois unités de bandes magnétiques. En outre, les échanges de données (entrée-sortie) peuvent se faire soit par un canal très rapide d'accès direct à la mémoire (un mot peut être acquis en un cycle de base dans les conditions limites), soit par des canaux d'accès programmés (un mot peut être acquis toutes les $40 \mu s$ environ).

II. L'équipement d'interface. - Il doit assurer l'acquisition et la transmission à quelques centaines de

( $\left.{ }^{1}\right)$ Expérience du groupe Crozon au Laboratoire de Physique Moléculaire du Collège de France. mètres des diverses informations qui apparaissent à une cadence fixée, soit par les événements de physique, soit par le rythme propre de l'accélérateur. Les premières sont délivrées à un instant quelconque au cours d'un temps de 0,5 s toutes les $3 \mathrm{~s}$ (cycle de Saturne) et conduisent à acquérir 70 à 80 mots par événement

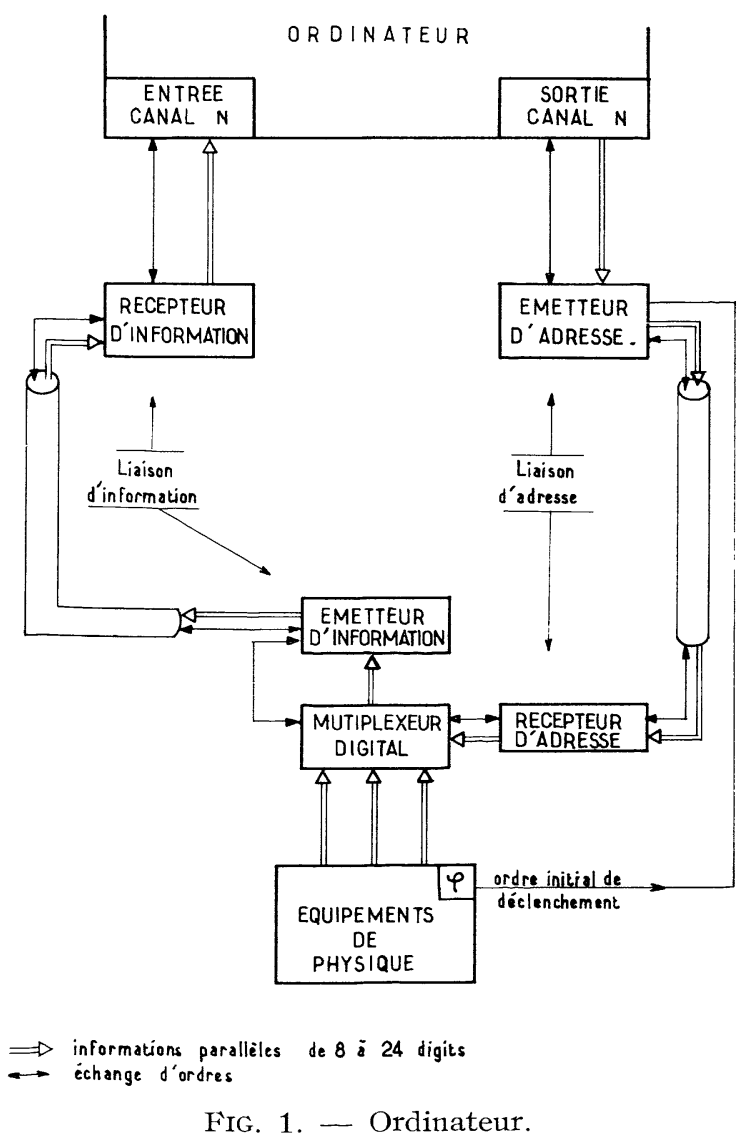

FIG. 1. - Ordinateur. 
en un temps inférieur à $1 \mathrm{~ms}$ (temps mort des chambres à fils), soit à une vitesse de l'ordre de $10^{5}$ mots/s. Les secondes sont disponibles une fois par cycle de l'accélérateur $(3 \mathrm{~s})$ et comprennent 16 mots acquis à la vitesse de $10^{3}$ mots/s.

Pour atteindre de telles vitesses d'acquisition, on a développé un système original ( $\left.{ }^{2}\right)$ fonctionnant sur câble coaxial à la vitesse de 20 mégabauds. La figure 1 donne le schéma de principe de l'un de ces systèmes qui comprend : i) Un multiplexeur digital chargé de collecter les diverses informations à acquérir. Il présente l'originalité d'être adressable et à longueur de mots variables modulés en octets (capacité nominale 64 octets); ii) Deux voies de transmission identiques constituées chacune d'un émetteur et d'un récepteur reliés par un câble coaxial unique. L'émetteur prend en compte les informations en parallèle (1 à 3 octets), les écoule en série sur le câble vers le récepteur qui les restitue en parallèle. La première voie transmet au multiplexeur un mot d'un octet délivré par l'ordinateur et représentant d'une part l'adresse du premier octet à acquérir ( 6 digits) et d'autre part le nombre d'octets (2 digits). Le multiplexeur acquiert l'information correspondante et la transmet à l'ordinateur par la seconde voie. Il s'écoule $6 \mu \mathrm{s}$ entre le moment où l'ordinateur fournit l'adresse et l'instant où il peut acquérir l'information correspondante (pour $100 \mathrm{~m}$ de distance). Tous ces équipements sont réalisés en circuits intégrés à la logique ECL.

III. La programmation. - Pendant le déroulement de l'expérience de physique, l'ordinateur remplit d'abord son rôle d'acquisition. Les informations en provenance des chambres à fils sont introduites dans l'ordinateur par le canal à accès direct à la mémoire; les contenus des 16 échelles lui parviennent par le canal programmé (type PIN-POT). A la fin de chaque cycle d'accélération, un bloc est constitué sur une bande magnétique qui servira par la suite à un traitement complet sur gros ordinateur. C'est ensuite la fonction de contrôle immédiat de l'expérience et de l'appareillage mis en jeu qu'effectue l'ordinateur. Ainsi,

(2) L'étude et la réalisation du système (Satris) ont été menées au C.E.N. Saclay par le Département Saturne (S.E.F.S.) en collaboration avec le Département d'Électronique Générale (S.I.N.). au début de chaque cycle, un « faux événement » de caractéristiques connues est transmis à l'ordinateur afin de vérifier aussi bien l'équipement d'interface que l'électronique de décodage associée aux chambres. En cas d'erreur, l'ordinateur émet un message et stoppe l'acquisition. Pour tout événement réel acquis, toute une série de vérifications est entreprise. En particulier, le fonctionnement de chaque chambre est vérifié individuellement, par le calcul de la vitesse de propagation du son dans le fil de magnétostriction. L'efficacité de chaque chambre est calculée automatiquement toutes les $10 \mathrm{mn}$ environ et sortie sur machine à écrire. Lorsque le fonctionnement des chambres est satisfaisant, des calculs plus élaborés sont entrepris pour reconstituer dans l'espace les trajectoires des trois particules. Des vérifications sont alors faites sur la position de l'apex, l'élasticité de la réaction, la corrélation angulaire et l'impulsion des protons de recul. Des alertes sont émises lorsque le nombre de défauts de chaque catégorie atteint une valeur prédéterminée par le physicien. L'ensemble de ces messages est transmis à la machine à écrire située sur le site même de l'expérience et à l'imprimante rapide proche de l'ordinateur qui tient ainsi le cahier d'expérience.

Enfin, à côté des programmes de traitement proprement dit, une partie importante de l'activité de l'ordinateur est consacrée au dialogue entre le physicien et l'ordinateur. A tout instant, le physicien peut, par une interruption externe, demander à dialoguer avec l'ordinateur par l'intermédiaire de la machine à écrire, soit pour obtenir des renseignements complémentaires, soit pour modifier certains paramètres. Ainsi un certain nombre d'histogrammes (vitesses, apex, coplanarité, etc.) sont accumulés sur l'ensemble des événements acquis et peuvent être édités à la demande sur l'imprimante. Un superviseur temps réel, qui a pour rôle essentiel de gérer l'ensemble des périphériques standards d'entrée-sortie, permet l'édition des messages sur ces périphériques en simultanéité avec l'acquisition et le traitement.

L'ensemble des programmes a été écrit en langage machine (Méta-symbol) afin d'obtenir une bonne efficacité aussi bien en encombrement mémoire qu'en temps d'exécution. L'encombrement de l'ensemble des programmes nécessaires à l'expérience est d'environ 15500 mots de 24 bits. L'unité centrale est utilisée pendant environ $20 \%$ du cycle de l'accélérateur. 